\title{
European Thyroid Association and Cardiovascular and Interventional Radiological Society of Europe 2021 Clinical Practice Guideline for the Use of Minimally Invasive Treatments in Malignant Thyroid Lesions
}

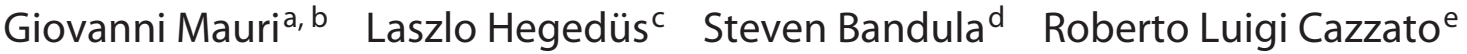 \\ Agnieszka Czarniecka ${ }^{f}$ Oliver Dudeck ${ }^{g}$ Laura Fugazzolah, i \\ Romana Netea-Maier ${ }^{j}$ Gilles Russk Göran Wallin' Enrico Papinim

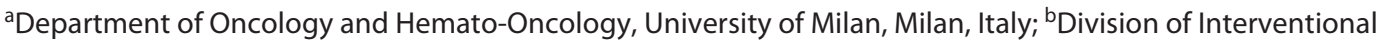 \\ Radiology, European Institute of Oncology, IRCCS, Milan, Italy; ' Department of Endocrinology, Odense University \\ Hospital, University of Southern Denmark, Odense, Denmark; Interventional Oncology Service, University College \\ Hospital, London, United Kingdom; 'Department of Interventional Radiology, University Hospital of Strasbourg,

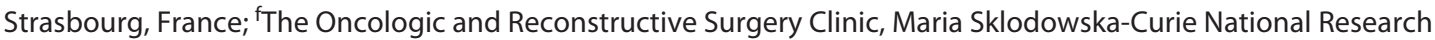 \\ Institute of Oncology, Gliwice Branch, Gliwice, Poland; ${ }^{9}$ Center for Microtherapy, Klinik Hirslanden, Zurich, \\ Switzerland; ' hepartment of Endocrinology and Metabolic Diseases, IRCCS Istituto Auxologico Italiano, Milan, Italy; \\ 'Department of Pathophysiology and Transplantation, University of Milan, Milan, Italy; 'Division of Endocrinology, \\ Department of Internal Medicine, Radboud University Medical Center, Nijmegen, The Netherlands; ${ }^{2}$ Thyroid and \\ Endocrine Tumors Unit, La Pitie-Salpetriere Hospital, Sorbonne University, Paris, France; 'Department of Surgery, \\ Faculty of Medicine and Health, Örebro University, Örebro, Sweden; mDepartment of Endocrinology \& Metabolism, \\ Ospedale Regina Apostolorum, Albano, Italy
}

\section{Keywords}

Thyroid cancer - Papillary thyroid microcarcinoma - Thyroid cancer metastases - Ultrasonography - Thermal ablation .

Microwaves · Laser therapy · Radiofrequency ablation

\begin{abstract}
The growing detection of papillary thyroid microcarcinomas (PTMCs) is paralleled by an increase in surgical procedures. Due to the frequent indolent nature, cost, and risk of surgery, active surveillance (AS) and ultrasound-guided minimally invasive treatments (MITs) are in suitable cases of incidental PTMC proposed as alternatives to thyroidectomy.
\end{abstract}

karger@karger.com www.karger.com/etj

Karger $\stackrel{\text { ' }}{5}$
Surgery and radioiodine are the established treatments for relapsing cervical differentiated thyroid carcinoma (DTC) metastases. But radioiodine refractoriness, risk of surgical complications, adverse influence on quality of life, or declining repeat surgery have led to AS and MIT being considered as alternatives for slow-growing DTC nodal metastases. Also, for distant radioiodine-refractory metastases not amenable to surgery, MIT is proposed as part of a multimodality therapeutic approach. The European Thyroid Association and the Cardiovascular and Interventional Radiological Society of Europe commissioned these guide-

Giovanni Mauri and Laszlo Hegedüs share first authorship.

Correspondence to:

Laszlo Hegedüs, laszlo.hegedus@ @rsyd.dk
(C) 2021 European Thyroid Association

Published by S. Karger AG, Basel 
lines for the appropriate use of MIT. Based on a systematic PubMed search, an evidence-based approach was applied, and both knowledge and practical experience of the panelists were incorporated to develop the manuscript and the specific recommendations. We recommend that when weighing between surgery, radioiodine, AS, or MIT for DTC, a multidisciplinary team including members with expertise in interventional radiology assess the demographic, clinical, histological, and imaging characteristics for appropriate selection of patients eligible for MIT. Consider TA in low-risk PTMC patients who are at surgical risk, have short life expectancy, relevant comorbidities, or are unwilling to undergo surgery or AS. As laser ablation, radiofrequency ablation, and microwave ablation are similarly safe and effective thermal ablation (TA) techniques, the choice should be based on the specific competences and resources of the centers. Use of ethanol ablation and high-intensity focused ultrasound is not recommended for PTMC treatment. Consider MIT as an alternative to surgical neck dissection in patients with radioiodine refractory cervical recurrences who are at surgical risk or decline further surgery. Factors that favor MIT are previous neck dissection, presence of surgical complications, small size metastases, and $<4$ involved latero-cervical lymph nodes. Consider TA among treatment options in patients with unresectable oligometastatic or oligoprogressive distant metastases to achieve local tumor control or pain palliation. Consider TA, in combination with bone consolidation and external beam radiation therapy, as a treatment option for painful bone metastases not amenable to other established treatments.

(C) 2021 European Thyroid Association Published by S. Karger AG, Basel

\section{Scope of the Document}

During the last decades, the incidence of differentiated thyroid cancer (DTC) is continuously rising in Europe and is estimated as high as 9 and 3 cases per 100,000 person-years in the female and male population, respectively [1]. Even if epidemiological differences are reported among different countries, papillary thyroid cancer (PTC) represents $80-85 \%$ and follicular cancer about $5-10 \%$ of cases, while medullary and anaplastic cancer account for the remaining [2]. Most DTCs are not aggressive tumors, as the disease-specific mortality rate is 0.7 and $0.5 \%$ per 100,000 person-years for women and men, respectively [1]. After successful initial treatment, local recurrences of PTC were registered during a 10-year follow-up in about $1.4 \%$ of patients and most of them occurred within 8 years [3]. However, the risk of recurrence or persistent structural disease rapidly increases in patients with advanced stage at diagnosis. Disease persistence or relapse are expected in $<5,6-20$, and $>20 \%$ of patients classified as low, intermediate, or high risk, respectively, according to the ATA risk of recurrence staging system [4].

So, the majority of DTCs are successfully cured with initial surgical treatment, followed, if needed, by radioiodine ablation (RAI) [4]. But, a few issues remain unsettled.

The growing incidental detection of papillary thyroid microcarcinomas (PTMCs) is paralleled by the progressive increase in surgical thyroid procedures [5]. Due to the frequently indolent nature of PTMC and the cost and risk of surgery, active surveillance (AS) and ultrasound (US)-guided minimally invasive treatments (MITs) are proposed as alternative management options to thyroidectomy for selected incidental PTMC [4, 6-8].

Moreover, after complete surgical resection, a followup by US may detect local recurrence in up to 7\% of DTC as a whole and in up to $2.4 \%$ of PTMC patients $[9,10]$. Repeat compartment-oriented surgery is the established therapeutic approach for DTC metastases which progressively grow during follow-up, but lymphadenectomy results in biochemical remission only in $21-61 \%$ of these patients $[11,12]$. Also, management problems may occur because of the absence of radioiodine uptake, the increased risk of surgical complications, the unfavorable influence of further surgery on quality of life, or the patient's declining repeat surgery after previous neck dissection. For these reasons, and the frequently indolent course of DTC lymph-node recurrences, current thyroid cancer guidelines suggest considering AS for cervical nodal metastases with a diameter $\leq 10 \mathrm{~mm}$ that appear as slow-growing [4]. As an alternative option, various image-guided MITs are now proposed as outpatient therapy of incidentally found indolent cervical DTC recurrences [13].

DTCs can result in distant metastases in up to $10 \%$ of cases, with lung and bone representing the 2 most commonly involved organs [14]. Various therapy options are currently available in metastatic patients, including RAI treatment, tyrosine-kinase inhibitors, surgery, radiation therapy (external beam radiation therapy [EBRT]), and MIT [15]. MIT is proposed in the multidisciplinary approach to patients with RAI refractory metastatic thyroid cancer who are fragile or not amenable to surgery, with the aim of achieving local control and/or pain palliation with minimal invasiveness of lung, bone, and liver disease. 
Currently, the major thyroid cancer guidelines dedicate only a marginal attention to the use of MIT for malignant thyroid disease management $[4,16]$. As the available information and the technical resources are rapidly evolving in this area [17], scope of the present guideline is to provide a clinical practice guide for the appropriate use of MIT, based on the best available scientific evidence and the authors' expertise.

\section{Methodology and Grading of Evidence}

The Executive Committees of the European Thyroid Association (ETA) and the Cardiovascular and Interventional Radiological Society of Europe (CIRSE) commissioned development of these guidelines to a task force led by 3 chairpersons (L.H., G.M., and E.P.). This team proposed a group of other 8 authors ( 4 from CIRSE and 4 from ETA) to compose the guideline panel. The proposal was based on clinical experience, scholarly approach, being an ETA or/a CIRSE member, and willingness to participate. The ETA and CIRSE guideline boards approved the composition of this team.

The relevant literature was taken into consideration using a systematic PubMed search. An evidence-based approach was applied, and both the knowledge and the practical experience of the panelists were incorporated to develop the manuscript and the specific recommendations. The Grading of Recommendations, Assessment, Development, and Evaluation system was used when rating the strength of the recommendations and the quality of evidence [18].

In accordance with previous guidelines, we used the following coding system: strong recommendation indicated by 1 and weak recommendation, or suggestion, indicated by 2 . The evidence grading is given as follows: very-low-quality (ØООО), low-quality (ØØОО), moderate-quality (ØØØО), and high-quality evidence $(\varnothing \varnothing \varnothing \varnothing)$. An electronic questionnaire was built on the Google Forms platform (Google LLC, Mountain View, CA, USA) and was disseminated to all participants and 2 Delphi rounds were performed to assess consensus.

Following discussions and after the task force had reached consensus, the draft was sent to the Guideline Boards for comments and thereafter posted on the ETA websites for 4 weeks for critical evaluation by the members. The received comments were evaluated by the authors and the guideline board, and the resulting changes were incorporated into the final document before submission to the European Thyroid Journal.

ETA-CIRSE Guideline on MIT in

Malignant Thyroid Lesions

\section{Techniques for Image-Guided Ablation of Thyroid Lesions: An Overview}

As a rule, before MIT, the diagnosis of DTC recurrence must be confirmed by fine needle aspiration or core-needle biopsy.Local anesthesia, with or without conscious sedation depending on the patients' wish, is preferred for the ablation of PTMC, cervical lymph-node metastases, and distant metastases in case of short procedural times. General anesthesia is preferred for the ablation of distant metastases requiring protracted treatment time (over 60 $\min )$.

\section{Ethanol Ablation}

Ethanol affects the tissues by inducing coagulative necrosis and endothelial damage resulting in thrombosis of small vessels, ischemic injury, and permanent tissue ablation. 21-27-gauge ( $\mathrm{G}$ ) needles are used, attached to a 1-5 $\mathrm{mL}$ syringe containing 95-99.9\% ethanol. Small amounts (0.1-0.5 mL) of ethanol are slowly injected, according to size and shape of the lesion to be treated, with constant US monitoring of the diffusion. Local anesthesia is generally used before ethanol ablation (EA). Immediately after the injection of ethanol, the treated area becomes echogenic on US, masking the needle tip and blurring the image of the area. After 1-3 min, the echogenic area decreases, and the needle tip is eventually repositioned into an untreated zone. Ethanol injections are repeated until the entire targeted tissue appears completely treated. Ethanol leakage outside the lesion may occur, resulting in severe pain and requiring the immediate cessation of injection [19-21].

\section{Thermal Ablation}

\section{Laser Ablation}

Laser is a focused beam of light energy, usually generated by a diode or a Nd:YAG source and delivered through an optical fiber into the target tissue [22-24]. In laser ablation (LA), the optical fibers are inserted through a $21 \mathrm{G}$ flexible guiding needle. The number of fibers to be inserted and the path to be followed are planned by the operator according to size and shape of the target lesion. Needle insertion along the major axis of the target lesion allows a repeat pullback for multiple illuminations. Injection of sterile saline or glucose solution around the target lesion can be performed to obtain a safety distance between the lesion and surrounding critical structures ("hydrodissection"). Hyperechoic signals, due to the formation of gas microbubbles, occurs during the ablation process, reflecting the heat-induced changes of the tissue. 
Most often, a fixed power protocol is used with LA, changing the total amount of energy delivered according to target tumor size and US modifications $[25,26]$.

\section{Radiofrequency Ablation}

An alternating electric current is applied to the target tissue via an electrode needle. The electric current rapidly oscillates tissue ions, creating frictional heating in areas of high current density adjacent to the electrode. Additional growth of the ablation zone is primarily due to thermal diffusion [13, 27]. Monopolar electrodes, with collecting pads and bipolar devices, without pads, are available $[27,28]$. Short (7-10 cm length) and thin (17-19 G) devices, appropriate for use in the neck region, are also available. Hydrodissection with a glucose solution can be used to increase the safety of the procedure. The electrode is introduced under US guidance into the target lesion, and in large lesions, a continuous repositioning of the applicator ("moving shot" technique) is performed to ablate the entire lesion $[27,28]$. The appearance of hyperechoic signals close to the electrode tip indicates the development of tissue changes, while a steep rise in tissue impedance demonstrates the achievement of tissue necrosis [27, 28].

\section{Microwave Ablation}

With microwave ablation (MWA), high-frequency ( $\mathrm{MHz}$ to $\mathrm{GHz}$ range) electromagnetic energy passes unimpeded into the tissue and induces hyperthermia by rapid oscillation of water molecules. This direct tissue heating is less susceptible to the perfusion cooling of blood supply, thereby allowing larger volume and more consistent tissue necrosis. The technique is otherwise identical to RFA, by means of probe introduction into the lesion under US guidance, followed by tumor coverage via multiple overlapping burns. Internally cooled antennas require a single low-loss cable and 14-17 G probes. Probe dimension is chosen according to the target tumor to be treated (either superficial neck lesions or deep liver/lung/ bone metastases) $[29,30]$.

\section{Cryoablation}

Localized tissue necrosis is achieved by inducing tissue hypothermia down to -40 to $-140^{\circ} \mathrm{C}$. Inside the needle probes, pressured gas (most frequently argon) passes through a narrow channel and is released within the device tip. Expansion of the gas causes rapid cooling via the Joule-Thompson effect, and an "ice ball" is formed in the adjacent tissue. Probes producing ice balls of various sizes are available which can be combined to produce a treat- ment volume which conforms to the target tumor. The ice ball is readily visible on computerized tomography and magnetic resonance and, less distinctly, with US examination, thus allowing intraprocedural monitoring - particularly useful when treating lesions close to critical structures. In cases at risk of complications, and for the protection of the skin in superficial tumors, hydrodissection should be preliminarily performed as for the heatrelated techniques.

The mechanism of tissue injury is incompletely understood, but osmotic insult, cell wall crystal injury, and microvascular thrombosis may all contribute to ablation. Unlike hyperthermia-induced necrosis, there is less disruption of the tissue architecture, partly reducing the risk of vascular and nerve injury, even if major complications may occur [31, 32]. This technique is generally less painful than heat-based technologies and is especially suitable for the treatment of superficial tumors on an outpatient basis.

The complication rate is not negligible after cryoablation. Pneumothorax, pleural effusion, and hemoptysis are reported in $61.7,70.5$, and $36.8 \%$ of lung cancer treatments [33]. In a recent series of 23 patients with painful sacral metastases treated with either RFA or cryoablation, no major complications occurred, but the frequency of minor complications was $23 \%$ [34].

\section{High-Intensity Focused Ultrasound Ablation}

High-intensity focused ultrasound ablation is the only completely noninvasive ablation technique. Ultrasonic energy, in the $20 \mathrm{kHz}-200 \mathrm{Mhz}$ range, induces molecular vibration, frictional heating, and cavitation. By focusing the energy from a probe outside the patient into a small volume, tissue destruction can be achieved within the target tumor without affecting intervening structures. Multiple overlapping ablation volumes may allow a complete target ablation. Care must be taken to avoid subtotal treatment because computerized control may prevent the ablation of peripheral areas close to critical structures $[35$, 36].

\section{Criteria for the Selection of Oncologic Patients Who Are Candidates for Minimally Invasive Treatment or a Multimodality Approach}

Indications for image-guided MITs in thyroid malignancies should be evaluated by a multidisciplinary team including experts in both traditional and image-guided interventional treatment modalities [13]. The choice of 
the appropriate MIT procedure, whether EA, LA, RFA, MWA, or high-intensity focused ultrasound ablation, depends on type and location of tumor, the team's experience, local technical resources, and the patients' characteristics [37]. Patients should always be informed about advantages and limitations of MIT in comparison with the other management options.

The use of MIT may be considered in patients with DTC in the following conditions:

1. PTMC

2. Unresectable thyroid cancer

3. Neck lymph-node recurrences of DTC

4. Distant metastases

In incidentally discovered low-risk PTMC in patients who are not eligible for or decline surgery, MIT may be considered as an alternative option to AS [3842]. A cytology suspicion of aggressive subtypes of thyroid carcinoma (e.g., tall cell, insular, and columnar cell), imaging detection of extrathyroidal growth or multiple neoplastic foci, presence of lymph node or distant metastases, and/or a worrisome molecular pattern (e.g., the detection of TERT promoter and TP53 mutations) contraindicate the use of MIT [38-42]. Moreover, due to the absence of comorbidities, higher risk of lymph node metastases and need of life-long follow-up, immediate surgery remains the best therapeutic approach to young subjects [40].

In patients with unresectable thyroid cancer, MIT should be considered as a palliative procedure for rapidly decreasing tumor burden and improving local symptoms, mainly in the context of multimodality treatment [43-45]. In patients with progressive RAI refractory cervical recurrences, who are at surgical risk or decline further surgery, MIT may be employed as an alternative to surgical neck dissection. MIT should be mainly considered in fragile patients with limited life expectancy, previous neck dissection, surgical complications, small size $(<20 \mathrm{~mm})$ metastases, and limited number $(<4)$ of involved lymph nodes in the latero-cervical compartments $[26,29,30,46,47]$. TA should be discouraged, except for palliative purposes, in patients with extensive lymph node involvement, central recurrences, evidence of radioiodine uptake, or clinical and histological factors suggesting an aggressive disease.

In selected patients with distant RAI refractory metastases from DTC, MIT may be proposed within the multimodality approach to advanced disease, with palliative intent (i.e., symptom relief) or in selected cases to achieve local tumor control. TA is, in particular, indicated in patients with bone metastases that are resistant to, or that could successively use, EBRT for preventing adverse skeletal events or for delaying systemic treatment $[48,49]$.

\section{Clinical Use in Thyroid Malignancies}

\section{Primary Thyroid Cancer}

MIT has been applied in the treatment of primary thyroid cancer, mainly in patients with incidentally diagnosed PTMC [40, 44, 50-54] and only rarely in patients with other types of primary thyroid tumors [43-45].

\section{Papillary Thyroid Microcarcinoma}

Due to the frequent indolent nature of PTMC, and the cost and risk of surgery, AS and MIT are considered as alternative management options to thyroidectomy for incidental PTMC $[8,55,56]$. Since the first feasibility study using laser for nonsurgical management of PTMC [57], US-guided treatments with laser, RFA, and MWA have been documented by several centers [40, 44, 50-54]. Three systematic reviews and meta-analyses have provided high-quality evidence of the efficacy and safety of RFA, MWA, and LA. The first study, based on 503 PTMCs with follow-up ranging from 8 to 53 months, demonstrated neither local tumor recurrence nor distant metastases after TA [6]. Cervical lymph node metastases were detected, at 24 and 30 months, respectively, in 2 patients (0.4\%). There were no major complications, and minor complications (transient dysphonia, intrathyroidal bleeding, and parenchymal edema) ranged from 3.4 to $19.0 \%$ [6]. A second meta-analysis, including 715 patients with followup ranging from 8 to 26 months, registered complete disappearance in $57.6 \%$ of treated PTMC. Scar-like remnants, devoid of viable cancer cells at FNA, were observed in the remaining cases. Major (persistent dysphonia) and minor complications were reported in 0.7 and $3.2 \%$ of cases, respectively, with a $0.4 \%$ local recurrence rate [7]. A third meta-analysis, analyzing results of RFA, MWA, and LA in 1,284 PTMCs, demonstrated that complete disappearance was the highest $(76.2 \%)$ and recurrence rate the lowest (0.01\%) after RFA, compared with the other 2 methods, although not statistically significant. Conversely, the complication rate was lower with LA $(0.9 \%)$ than with RFA (1.7\%) and MWA (6.0\%). No distant metastases occurred during the 7.8 months median follow-up [41]. Notably, in the reported meta-analysis, the followup was generally short for thyroid cancer surveillance, and the authors never mentioned the thyroglobulin level. Randomized head-to-head trials of TA versus surgery are 
lacking but recent studies report the outcomes of large retrospective series. Ninety-four patients treated with RFA were compared to 80 patients treated surgically during the same period [58]. After 5 years of follow-up, clinical outcomes were similar but procedure time, hospital stay, and cost of RFA were lower than those with surgery. The surgery group had significantly more major complications and lower posttreatment thyroid-related quality of life. In another study, 81 patients were treated with either surgery or LA with a 49-month mean follow-up; $94.4 \%$ of the ablated lesions completely disappeared and $5.6 \%$ remained as scar-like zones. Complication rates and recurrence rates did not differ significantly between the LA (2.8 and 5.6\%, respectively) and the surgical group (6.7 and 6.7\%, respectively). Mean hospital stay and procedure time were also shorter in the LA group. No distant metastases occurred in either group [59]. Robust comparative data are available in 2 meta-analyses. The first, including 867 patients, demonstrated that recurrence rate and recurrence-free survival following TA and surgery were similar, but that TA had a significantly lower complication rate (odds ratio [OR] 0.24), postoperative length of stay, and costs (pooled standard mean difference -1.69) [60]. The second meta-analysis, involving 1,289 patients, demonstrated that the risk of hoarseness (OR 0.33), late hypothyroidism (OR 0.31), and postoperative pain (OR 0.35 ) was lower and the hospitalization time was shorter (SMD -4.01) in the TA group. The TA group also obtained a better cosmetic result while other symptomatology did not differ significantly compared to surgery [61].

EA was employed from 2010 to 2017, in a small series of 15 patients with PTMC. After ethanol injection, tumor shrinkage and absence of Doppler flow was demonstrated in all cases. Neither major complications nor local recurrence or nodal metastases were reported [62].

AS has been proposed as an alternative to immediate surgery for PTMC, balancing the low probability of cancer progression, with prevention of surgical complications and late hypothyroidism [55]. No head-to-head comparison of TA versus AS is available, but the advantage of the ablation of the neoplastic area is potentially relevant. During a 5-year AS period, linear growth of PTMC volume has been reported in $12-23 \%$ of cases, while a minority of patients, especially among the young, developed extrathyroidal spread and cervical metastases after several years of observation [56, 63, 64]. Finally, as from 8.7 to $32.0 \%$ of patients in AS programs undergo delayed surgery for reasons unrelated to cancer growth [6], TA could also prevent the anxiety caused by awareness of harboring a tumor which may grow and eventu- ally need more aggressive surgical removal [65]. The main advantages of TA are the possibility of offering an active treatment, which seems to be as effective as surgical removal, in an outpatient setting, with reduced invasiveness, no need of general anesthesia, and low risk of complications and hypothyroidism [17].

\section{Unresectable Thyroid Cancer}

A different scenario is represented by using MIT for tumor volume reduction and control of threatening local symptoms in unresectable primary DTC, medullary, or anaplastic carcinomas. Few data are available on this topic, but a mean volume reduction of $51 \%$ was observed in a series of inoperable DTCs. Importantly, RFA was not effective if the tumors had overt vascular encasement or massive calcification due to the difficult approach and the insufficient burden ablation [66]. In a large series of DTCs invading the airways, the volume reduction rate was $81.2 \%$ and complete disappearance rate, $72.1 \%$ (mean follow-up, 47.9 months). The efficacy decreased significantly according to the degree of tracheal abutment of the tumors [35].

Both LA and RFA may be used for the management of medullary thyroid cancer in patients not amenable to surgery $[67,68]$. A few case reports demonstrated the efficacy of TA for short-term local control of medullary thyroid cancer, but the available evidence is still limited.

The feasibility of LA has been also reported for some anaplastic thyroid cancers, usually with minor short-term effects on symptoms and without effects on thyroid volume reduction [43-45]. Thus, treatment of inoperable anaplastic or medullary thyroid carcinoma with TA is characterized by inconsistent outcomes, and it should be considered only in patients with no other possible option, with palliative intent. In these cases, MIT may be part of a multimodality approach to advanced thyroid cancer but should never delay the use of systemic therapy and EBRT for this deadly disease (Tables 1,2).

\section{Follicular Neoplasms}

Few reports consider the use of TA for thyroid nodules with a cytological diagnosis of follicular neoplasm. In a 5 -year follow-up study, 8 of 10 follicular lesions $<2.0 \mathrm{~cm}$ in largest diameter completely disappeared, and 2 showed $97 \%$ volume reduction after RFA. There were no recurrences or distant metastases during the follow-up period [69]. In a second study, 6 thyroid lesions with follicular cytology and negative BRAF and NRAS molecular testing were treated with RFA and followed up until 24 months [70]. Two lesions regrew after treatment and were surgi- 
Table 1. Indications for use of MITs in PTMCs

\begin{tabular}{ll}
\hline Factors favoring thermal ablation & Factors favoring surgery \\
\hline Demographics & Demographics \\
Old age & Young age \\
Relevant comorbidities & No comorbidities \\
No family history of aggressive forms & Familial form \\
Contralateral vocal cord palsy & - \\
Refusal of surgery & - \\
\hline Cytology & Cytology \\
Papillary carcinoma classical variant & Worrisome cytology features \\
- & High-risk molecular pattern \\
\hline US examination & US examination \\
Central location & Subcapsular location \\
Well defined margins & Posterior location \\
Absence of capsular contact & Paratracheal location \\
Solitary thyroid lesion & Multinodular goiter \\
No evidence of extrathyroidal spread & Extrathyroidal spread \\
\hline Technical resources & Technical resources \\
Expertise in US-guided ablation procedures & High-volume thyroid surgery \\
\hline
\end{tabular}

MITs, minimally invasive treatments; PTMCs, papillary thyroid microcarcinomas; US, ultrasound.

cally excised. Histological examination revealed a follicular carcinoma and a follicular neoplasm of indeterminate malignant behavior. Therefore, surgery remains the established standard of care for the management of thyroid nodules with Bethesda IV cytology because of the risk of delayed diagnosis, and distant metastasis, in those follicular lesions that correspond to follicular thyroid cancers. TA should be restricted to patients with follicular lesions who are at surgical risk and present favorable US features and negative molecular testing as an alternative to clinical surveillance.

\section{Neck Recurrences of Thyroid Cancer}

The majority of DTCs are successfully cured with initial surgical treatment, followed, if needed, by RAI ablation [15]. Even after apparently successful treatment, recurrence can occur in the thyroid bed and/or as nodal metastases. Repeat compartment-oriented surgery is the established therapeutic approach for DTC recurrences but lymphadenectomy results in biochemical and structural remission only in some of these patients [71]. Thus, AS and MIT can be considered an alternative to surgery for selected patients with cervical DTC metastases [15].

EA has been reported as effective in local disease control in a few single-center case series $[19,72,73]$. The largest retrospective study, including 109 neck lymph node metastases, with a 38-month median follow-up, demon- strated complete response in $84 \%$ [19]. Up to 5 EA sessions were needed for complete ablation and, despite reporting no major complications, local pain and side effects may occur frequently. The major limitations of EA are the uneven and unpredictable area of ablation and risk of ethanol seeping into neck tissues [8]. A retrospective study comparing the outcomes of 42 patients with recurrence of DTC, treated either with EA or RFA, found no recurrences in the RFA group (61 months follow-up), while 5 of 21 (23\%) metastases had local progression at 4-11 months after EA [74]. Retrospective and prospective studies have demonstrated efficacy of RFA, LA, and MWA for local control of DTC recurrence $[29,47,75,76]$. A 2016 meta-analysis, including 255 metastatic lesions treated with US-guided RFA, demonstrated a significant tumor volume decrease at 6 months follow-up [77]. A second meta-analysis, evaluating the efficacy and safety of RFA and EA for treating locally recurrent thyroid cancer, included 415 thyroid lesions [78]. The complete disappearance rate after RFA was $68.8 \%$ and the recurrence rate, $0.0 \%$, with a complication rate of $1.6 \%$.

Head-to-head prospective studies of TA versus surgery are unavailable, but a few papers retrospectively compared the outcomes of RFA and surgical neck dissection. In a study in patients with $<20$-mm cervical DTC recurrences, 27 treated with RFA and 46 treated with surgery, the 3-year recurrence-free survival was similar in 
Table 2. Summary of recommendations

Summary of recommendations

Recommendation 1

Consider the use of image-guided MITs in the multimodal approach to patients with thyroid cancer. Strength of recommendation 1; evidence ØØOO. Strong agreement $(11 / 11,100 \%)$

Recommendation 2

A multidisciplinary team, which includes members with specific expertise in MIT, perform the selection of patients eligible for MIT based on the patient's clinical, demographic, and imaging characteristics. Strength of recommendation 1; evidence $\varnothing \varnothing O O$. Strong agreement $(11 / 11,100 \%)$

Recommendation 3

Select the MIT modality based on involved organ(s), staging of the disease, patient characteristics and preferences, and specific competences and resources at the treating center. Strength of recommendation 1; evidence ØØOO. Strong agreement (11/11, 100\%)

Recommendation 4

Inform patients about MIT feasibility and its advantages and limitations in comparison with the other management strategies.

Strength of recommendation $1 *$; evidence ØOOO. Strong agreement $(11 / 11,100 \%)$

Recommendation 5

Consider the use of image-guided TA for patients with low-risk PTMC, mainly if the patient is at surgical risk, is expected to have short life expectancy, has comorbidities that need to be prioritized before thyroid surgery, or is unwilling to undergo surgery or AS. Strength of recommendation 1; evidence ØØOO. Strong agreement $(11 / 11,100 \%)$

Recommendation 6

Inform patients with incidentally discovered PTMC who are suitable for AS about TA as a therapeutic alternative to immediate surgery or AS. Strength of recommendation $1^{*}$, evidence $\varnothing$ OOO. Strong agreement $(11 / 11,100 \%)$

Recommendation 7

Inform patients with PTMC about advantages and limitations of TA in comparison with the other management options and the need of protracted clinical and US follow-up for ruling out potential loco-regional recurrences. Strength of recommendation 1; evidence Ø๐OO. Strong agreement $(11 / 11,100 \%)$

Recommendation 8

Choose treatment modality based on specific competences and resources at the individual center, because LA, RFA, and MWA are similarly safe and effective TA techniques for low-risk PTMC. Strength of recommendation 1; evidence ØØOO. Strong agreement $(11 / 11 ; 100 \%)$

Recommendation 9

Abstain from using EA and HIFU for PTMC treatment, due to insufficient evidence and technical limitations. Strength of recommendation $1^{*}$; evidence $\varnothing$ OOO. Strong agreement $(11 / 11,100 \%)$

Recommendation 10

Consider MIT for palliative purposes, preferentially in the context of a multimodality approach, in patients with primary thyroid cancer, other than low-risk PTMC. Strength of recommendation 2*; evidence ØOOO. Strong agreement $(11 / 11,100 \%)$

Recommendation 11

Consider MIT as an alternative option to surgical neck dissection in patients with radioiodine refractory cervical recurrences who are at surgical risk or decline further surgery. Strength of recommendation 1; evidence ØØOO. Strong agreement (11/11, 100\%)

Recommendation 12

Confirm the diagnosis of DTC recurrence by fine needle aspiration or core-needle biopsy before MIT. Strength of recommendation 1; evidence ØØOO. Strong agreement $(11 / 11,100 \%)$

Recommendation 13

Consider the following clinical factors as favoring MIT for DTC: cervical recurrences, a previous surgical neck dissection, presence of surgical complications, small size metastases, and limited latero-cervical lymph node involvement. Strength of recommendation 1 ; evidence ØØOO. Strong agreement $(11 / 11,100 \%)$

Recommendation 14

Consider MIT only for palliative purposes in DTC recurrences with extensive lymph node involvement, central location, evidence of radioiodine uptake, and clinical and histological factors suggestive of aggressive disease. Strength of recommendation 1; evidence ØØОO. Strong agreement $(11 / 11,100 \%)$ 
Table 2 (continued)

Summary of recommendations

Recommendation 15

Consider TA among the treatment options in patients with oligometastatic or oligoprogressive disease, to achieve local tumor control or pain palliation. Strength of recommendation 1; evidence ØØOO. Strong agreement $(11 / 11,100 \%)$

Recommendation 16

Consider TA for the palliative treatment of painful bone metastases. Ablation may be proposed alone or in combination with bone consolidation and EBRT to improve the clinical outcome. Strength of recommendation 1; evidence ØØOO. Strong agreement (11/11, $100 \%)$

Recommendation 17

Consider TA, alone or in combination with EBRT, with a local curative intent for $<20 \mathrm{~mm}$ bone metastases. Strength of recommendation $2 *$, evidence ØOOO. Strong agreement $(11 / 11,100 \%)$

Recommendation 18

Consider TA for residual RAI-refractory oligo-metastatic lung (sized $<2 \mathrm{~cm}$ ) and liver (sized $<3 \mathrm{~cm}$ ) DTC metastases, preferentially in the context of a multimodality approach. Strength of recommendation 1; evidence ØOOO. Strong agreement (11/11, 100\%)

MITs, minimally invasive treatments; TA, thermal ablation; PTMC, papillary thyroid microcarcinoma; AS, active surveillance; US, ultrasound; LA, laser ablation; RFA, radiofrequency ablation; MWA, microwave ablation; EA, ethanol ablation; HIFU, high-intensity focused ultrasound; DTC, differentiated thyroid carcinoma; EBRT, external beam radiation therapy. ${ }^{*}$ The strength of this recommendation was considered as appropriate by the panel of experts though, for evident reasons, the evidence level was not based on prospective randomized setting.

the 2 groups ( 96.0 and $92.6 \%$, respectively). Major complications, mostly hypocalcemia, occurred only in the reoperated group [76]. In a long-term retrospective study, with propensity score adjustment, 2 cohorts of 70 patients with locally recurrent thyroid cancer underwent either RFA or repeat surgery. The recurrence-free survival rates in the RFA and in the surgery groups were similar (89.4 vs. $94.5 \%$ at 6 years, respectively) with no significant differences in mean posttreatment serum Tg levels. Hypocalcaemia and overall complication rates were significantly higher in the surgery group (7 cases in the RFA vs. 27 cases in the surgery group) [79].

LA was reported feasible in RAI refractory cervical DTC recurrence following cervical lymph-node dissection. A prospective trial in 8 non-RAI-avid cervical recurrences demonstrated a mean volume decrease from 0.64 to $0.07 \mathrm{~mL}$ at 12 -months, with undetectable Tg levels in $60 \%$ of cases [75]. In 46 18FDG-PET/computerized tomography-positive nodal metastases followed up for 30 months [46], local control was obtained in 40 of 46 (86.9\%) lymph nodes, with no detectable residual disease in $79 \%$ of patients.

Also, efficacy of LA was demonstrated in a patient with medullary thyroid cancer recurrence [26]. In a retrospective study of 24 lymph node metastases of PTC treated with MWA, nearly complete disappearance was achieved with no late recurrence reported during a 32-month fol- low-up [30]. A prospective study of 23 cervical PTC recurrences, with an up to 18-month follow-up, demonstrated a $91 \%$ mean volume reduction at the final followup. Complete disappearance in $30.4 \%$ and persistence as scar-like areas in $52.2 \%$ were reported. One patient experienced transient dysphonia, but no permanent complications occurred [29].

Notably, TA of thyroid cancer metastases is followed by a rapid increase of serum Tg levels during the following $48 \mathrm{~h}$. After complete destruction of lymph node metastases, serum Tg progressively decreases to undetectable levels and becomes a sensitive blood marker for monitoring potential disease relapse $[25,75]$. Similarly, serum calcitonin level may be used as an effective marker for monitoring the local control of medullary thyroid cancer metastases after thermal ablation of nodal recurrences [26].

\section{Distant Metastases}

Follicular and, to a lesser extent, papillary thyroid carcinomas result in distant metastases in up to $10 \%$ of cases, with lung and bone representing the 2 most commonly involved organs [14]. Various therapy options are currently available in metastatic patients, including RAI treatment, tyrosine-kinase inhibitors, surgery, EBRT, and MIT [15]. MIT is proposed in the multidisciplinary approach to patients with RAI refractory metastatic thyroid 
cancer who are fragile or not amenable to surgery. The aim being local disease control in oligometastatic or oligoprogressive patients and/or pain palliation with minimal invasion. However, outcome evidence is limited and of modest quality.

\section{Lung Metastases}

RFA treatment of 1,037 lung metastases from primary cancers of colon, rectum, kidney, and thyroid (the latter accounting for only $3 \%$ ), treated up to 4 times without major complications, demonstrated progression-free survival and local tumor progression rates of 40.2 and $13.1 \%$, and 5.9 and $11.0 \%$, at 1 and 4 years, respectively [80]. Factors associated with poor overall and progression-free survival were primary disease other than colon or kidney, disease-free interval $\leq 1$-year, tumor size $>20$ $\mathrm{mm}$, and $>2$ pulmonary metastases. Pneumothorax was the most common adverse event, occurring in $67 \%$ of ablation procedures and requiring treatment in $28 \%$ of cases, while the 30 -day mortality was $0.35 \%$.

\section{Bone Metastases}

The most common site of bone metastases from thyroid cancer is the spine, severely affecting the quality of life and frequently resulting in focal neurologic symptoms and pathologic fractures. Treatment options, besides repeated RAI therapy in cases with persistent radioiodine uptake, include surgical resection, vertebroplasty, EBRT, and MIT. In a single-arm prospective multicenter study [81], RFA significantly relieved pain intensity from bone metastases at the 3-month follow-up. Three major adverse effects (refractory pain and neural damages) were reported. Similarly, a study using cryoablation [82] reported a mean score for worst pain in a 24 -h period decreasing from 7.1/10 before treatment to $5.1 / 10$ and $1.4 / 10$ at 1 - and 24 -week follow-up, respectively; $83 \%$ of patients reporting opioid use before cryoablation reduced their intake during follow-up, and only 1 case of infection was registered. LA was employed for the management of 6 DTC metastatic lesions resistant to high-dose RAI therapy, followed by EBRT and on opioid analgesics [83]. At 6 months, imaging showed massive reduction of tumor burden associated with a significant decrease in all pain variables. No major complications occurred, and daily opioid requirement significantly decreased. Secondary fractures represent the most common major adverse event following bone tumor ablation $[84,85]$. Therefore, TA should be combined, especially for weight-bearing bones, with bone consolidation by osteoplasty or osteosynthesis [86, 87].

\section{Liver Metastases}

There is considerable experience with TA of liver lesions, including metastases from different tumors [88, 89]. After RFA in 512 colorectal liver metastases, local tumor progression-free and overall survival were 72 and $30 \%$ at 10 years, and 72 and $28 \%$ at 10 and 15 years, respectively [89]. Small ablation margins $(<5 \mathrm{~mm})$ and large tumor size $(>2 \mathrm{~cm})$ were factors affecting complete ablation and local tumor progression $[88,89]$. Importantly, major complications following MIT were rare (1-7\%) $[88,89]$, and percutaneous TA was safely combined with systemic treatments. Only sporadic reports are available on TA outcomes in liver metastases of other origins, including thyroid cancer. Use of LTA and RFA were described in single case-reports $[90,91]$ as effective for rapid tumor burden decrease and associated with marked, but temporary, symptom improvement. Therefore, TA should be considered in a multimodality approach to DTC patients who are at surgical risk and in whom other treatment modalities are ineffective. Anecdotal reports are also available for RFA treatment of liver metastases from medullary thyroid cancer [67].

\section{Concluding Remarks}

The major hindrances before implementing these technologies in the treatment of malignant thyroid disease are the same as those for benign thyroid disease, which are primarily, lack of guidelines and access to these technologies for thyroid disease $[92,93]$. Realizing this has been among the main drivers of the present guideline and the ambition to establish teaching courses in the near future.

\section{Conflict of Interest Statement}

G.M.: consultancy from Elesta SrL, speaking fee from GE, Advisory Board for Boston Scientific. All other authors declare they have no conflicts of interest to declare.

\section{Funding Sources}

No funds were received for the present work.

\section{Author Contributions}

All authors contributed to the idea, gathered the information, interpreted the data, and wrote and accepted the final version of the manuscript. 


\section{References}

1 European Network of Cancer Registries Factsheets [Internet]. [cited 2021 Apr 7]. Available from: https://www.encr.eu/sites/default/ files/factsheets/ENCR_Factsheet_Thyroid_2017-2.pdf.

2 Figge JJ. Epidemiology of thyroid cancer. In: Thyroid cancer (second edition): a comprehensive guide to clinical management. Totowa, NJ: Humana Press; 2006. p. 9-13.

3 Durante C, Montesano T, Torlontano M, Attard M, Monzani F, Tumino S, et al. Papillary thyroid cancer: time course of recurrences during postsurgery surveillance. J Clin Endocrinol Metab. 2013 Feb;98(2):636-42.

4 Haugen BR, Alexander EK, Bible KC, Doherty GM, Mandel SJ, Nikiforov YE, et al. 2015 American thyroid association management guidelines for adult patients with thyroid nodules and differentiated thyroid cancer: the American thyroid association guidelines task force on thyroid nodules and differentiated thyroid Cancer. Thyroid. 2016 Jan;26(1):1133.

5 Lim H, Devesa SS, Sosa JA, Check D, Kitahara CM. Trends in thyroid cancer incidence and mortality in the United States, 1974-2013. JAMA. 2017 Apr;317(13):1338-48.

6 Cho SJ, Baek JH, Chung SR, Choi YJ, Lee JH. Thermal ablation for small papillary thyroid cancer: a systematic review. Thyroid. 2019 Dec;29(12):1774-83.

7 Choi Y, Jung S-L. Efficacy and safety of thermal ablation techniques for the treatment of primary papillary thyroid microcarcinoma: a systematic review and meta-analysis. Thyroid. 2020 May;30(5):720-31.

8 Mauri G, Orsi F, Carriero S, Della Vigna P, De Fiori E, Monzani D, et al. Image-guided thermal ablation as an alternative to surgery for papillary thyroid microcarcinoma: preliminary results of an Italian experience. Front Endocrinol. 2021;11:575152.

9 Chereau N, Oyekunle TO, Zambeli-Ljepović A, Kazaure HS, Roman SA, Menegaux F, et al. Predicting recurrence of papillary thyroid cancer using the eighth edition of the AJCC/ UICC staging system. Br J Surg. 2019 Jun; 106(7):889-97.

10 Roti E, degli Uberti EC, Bondanelli M, Braverman LE. Thyroid papillary microcarcinoma: a descriptive and meta-analysis study. Eur J Endocrinol. 2008 Dec;159(6):659-73.

11 Urken ML, Milas M, Randolph GW, Tufano $\mathrm{R}$, Bergman D, Bernet V, et al. Management of recurrent and persistent metastatic lymph nodes in well-differentiated thyroid cancer: a multifactorial decision-making guide for the thyroid cancer care collaborative. Head Neck. 2015 Apr;37(4):605-14.

12 Al-Saif O, Farrar WB, Bloomston M, Porter $\mathrm{K}$, Ringel MD, Kloos RT. Long-term efficacy of lymph node reoperation for persistent papillary thyroid cancer. J Clin Endocrinol Metab. 2010;95(5):2187-94.
13 Mauri G, Gennaro N, Lee MK, Baek JH. Laser and radiofrequency ablations for benign and malignant thyroid tumors. Int J Hyperthermia. 2019 Oct;36(2):13-20.

14 Durante C, Haddy N, Baudin E, Leboulleux S, Hartl D, Travagli JP, et al. Long-term outcome of 444 patients with distant metastases from papillary and follicular thyroid carcinoma: benefits and limits of radioiodine therapy. J Clin Endocrinol Metab. 2006;91(8): 2892-9.

15 Haugen BR, Alexander EK, Bible KC, Doherty GM, Mandel SJ, Nikiforov YE, et al. 2015 American thyroid association management guidelines for adult patients with thyroid nodules and differentiated thyroid cancer: the American thyroid association guidelines task force on thyroid nodules and differentiated thyroid cancer. Thyroid. 2016 Jan;26(1):1133.

16 Filetti S, Durante C, Hartl D, Leboulleux S, Locati LD, Newbold K, et al. Thyroid cancer: ESMO clinical practice guidelines for diagnosis, treatment and follow-up. Ann Oncol. 2019 Dec;30(12):1856-83.

17 Hegedüs L, Miyauchi A, Tuttle RM. Nonsurgical thermal ablation of thyroid nodules: not if, but why, when, and how? Thyroid. 2020 Dec;30(12):1691-4.

18 Swiglo BA, Murad MH, Schünemann HJ, Kunz R, Vigersky RA, Guyatt GH, et al. A case for clarity, consistency, and helpfulness: stateof-the-art clinical practice guidelines in endocrinology using the grading of recommendations, assessment, development, and evaluation system. J Clin Endocrinol Metab. 2008; 93(3):666-73.

19 Heilo A, Sigstad E, Fagerlid KH, Håskjold OI, Grøholt KK, Berner A, et al. Efficacy of ultrasound-guided percutaneous ethanol injection treatment in patients with a limited number of metastatic cervical lymph nodes from papillary thyroid carcinoma. J Clin Endocrinol Metab. 2011 Sep;96(9):2750-5.

20 Hahn SY, Shin JH, Na DG, Ha EJ, Ahn HS, Lim HK, et al. Ethanol ablation of the thyroid nodules: 2018 consensus statement by the Korean society of thyroid radiology. Korean J Radiol. 2019 Apr;20(4):609-20.

21 Jeong SY, Baek JH, Choi YJ, Lee JH. Ethanol and thermal ablation for malignant thyroid tumours. Int J Hyperthermia. 2017 Dec;33(8): 938-45.

22 Gharib H, Hegedüs L, Pacella CM, Baek JH, Papini E. Clinical review: nonsurgical, imageguided, minimally invasive therapy for thyroid nodules. J Clin Endocrinol Metab. 2013 Oct;98(10):3949-57.

23 Papini E, Pacella CM, Misischi I, Guglielmi R, Bizzarri G, Døssing H, et al. The advent of ultrasound-guided ablation techniques in nodular thyroid disease: towards a patient-tailored approach. Best Pract Res Clin Endocrinol Metab. 2014;28(4):601-18.
24 Mauri G, Nicosia L, Della vigna P, Varano GM, Maiettini D, Bonomo G, et al. Percutaneous laser ablation for benign and malignant thyroid diseases. Ultrasonography. 2019 Jan; 38(1):25-36.

25 Mauri G, Cova L, Tondolo T, Ierace T, Baroli A, Di Mauro E, et al. Percutaneous laser ablation of metastatic lymph nodes in the neck from papillary thyroid carcinoma: preliminary results. J Clin Endocrinol Metab. 2013 Jul;98(7):E1203-7.

26 Persichetti A, Bizzarri G, Guglielmi R, Barnabei A, Bianchini A, Coccaro C, et al. Ultrasound-guided laser ablation for local control of neck recurrences of medullary thyroid cancer. A feasibility study. Int J Hyperthermia. 2018 Dec;35(1):480-92.

27 Park HS, Baek JH, Park AW, Chung SR, Choi YJ, Lee JH. Thyroid radiofrequency ablation: updates on innovative devices and techniques. Korean J Radiol. 2017;18(4):615-23.

28 Kim JH, Baek JH, Lim HK, Ahn HS, Baek SM, Choi YJ, et al. 2017 Thyroid radiofrequency ablation guideline: Korean society of thyroid radiology. Korean J Radiol. 2018;19(4):632-55.

29 Yue W, Chen L, Wang S, Yu S. Locoregional control of recurrent papillary thyroid carcinoma by ultrasound-guided percutaneous microwave ablation: a prospective study. Int J Hyperthermia. 2015 Jun;31(4):403-8.

30 Teng D, Ding L, Wang Y, Liu C, Xia Y, Wang $\mathrm{H}$. Safety and efficiency of ultrasound-guided low power microwave ablation in the treatment of cervical metastatic lymph node from papillary thyroid carcinoma: a mean of 32 months follow-up study. Endocrine. 2018 Dec;62(3):648-54.

31 Cazzato RL, Garnon J, Ramamurthy N, Koch G, Tsoumakidou G, Caudrelier J, et al. Percutaneous image-guided cryoablation: current applications and results in the oncologic field. Med Oncol. 2016;33(12):1-16.

32 Erinjeri JP, Clark TWI. Cryoablation: mechanism of action and devices. J Vasc Interv Radiol. 2010 Aug;21(Suppl 8):S187-91.

33 Inoue $\mathrm{M}$, Nakatsuka S, Yashiro H, Ito N, Izumi Y, Yamauchi Y, et al. Percutaneous cryoablation of lung tumors: feasibility and safety. J Vasc Interv Radiol. 2012 Mar;23(3):295-305.

34 Cazzato RL, De Marini P, Leonard-Lorant I, Dalili D, Koch G, Autrusseau PA, et al. Percutaneous thermal ablation of sacral metastases: assessment of pain relief and local tumor control. Diagn Interv Imaging. 2021 Apr;31(4):2153-60.

35 Chung SR, Baek JH, Choi YJ, Sung TY, Song DE, Kim TY, et al. Efficacy of radiofrequency ablation for recurrent thyroid cancer invading the airways. Eur Radiol. 2021 Apr; 31(4): 2153-60.

36 Orgera G, Monfardini L, Della Vigna P, Zhang L, Bonomo G, Arnone P, et al. Highintensity focused ultrasound (HIFU) in patients with solid malignancies: evaluation of feasibility, local tumour response and clinical results. Radiol Med. 2011 Aug;116(5):734-48.
ETA-CIRSE Guideline on MIT in

Malignant Thyroid Lesions
Eur Thyroid J 2021;10:185-197

DOI: $10.1159 / 000516469$ 
37 Dietrich CF, Müller T, Bojunga J, Dong Y, Mauri G, Radzina M, et al. Statement and recommendations on interventional ultrasound as a thyroid diagnostic and treatment procedure. Ultrasound Med Biol. 2018 Jan;44(1):14-36.

38 Zhang M, Luo Y, Zhang Y, Tang J. Efficacy and safety of ultrasound-guided radiofrequency ablation for treating low-risk papillary thyroid microcarcinoma: a Prospective Study. Thyroid. 2016 Nov;26(11):1581-7.

39 Ding M, Tang X, Cui D, Chi J, Shi Y, Wang T, et al. Clinical outcomes of ultrasound-guided radiofrequency ablation for the treatment of primary papillary thyroid microcarcinoma. Clin Radiol. 2019;74(9):712-7.

40 Lim HK, Cho SJ, Baek JH, Lee KD, Son CW, Son JM, et al. US-guided radiofrequency ablation for low-risk papillary thyroid microcarcinoma: efficacy and safety in a large population. Korean J Radiol. 2019 Dec;20(12):1653-61.

41 Tong M, Li S, Li YY, Li YY, Feng Y, Che Y. Efficacy and safety of radiofrequency, microwave and laser ablation for treating papillary thyroid microcarcinoma: a systematic review and meta-analysis. Int J Hyperthermia. 2019 Jan;36(1):1278-86.

42 Cho SJ, Baek SM, Lim HK, Lee KD, Son JM, Baek JH. Long-term follow-up results of ultrasound-guided radiofrequency ablation for low-risk papillary thyroid microcarcinoma: more than 5 year follow-up for 84 tumors. Thyroid. 2020 Dec;30(12):1745-51.

43 Cakir B, Topaloglu O, Gul K, Agac T, Aydin C, Dirikoc A, et al. Ultrasound-guided percutaneous laser ablation treatment in inoperable aggressive course anaplastic thyroid carcinoma: the introduction of a novel alternative palliative therapy: second experience in the literature. J Endocrinol Invest. 2007;30(7): 624-5.

44 Jeong SY, Baek JH, Choi YJ, Chung SR, Sung TY, Kim WG, et al. Radiofrequency ablation of primary thyroid carcinoma: efficacy according to the types of thyroid carcinoma. Int J Hyperthermia. 2018 Jul;34(5):611-6.

45 Pacella CM, Bizzari G, Spiezia S, Bianchini A, Guglielmi R, Crescenzi A, et al. Thyroid tissue: US-guided percutaneous laser thermal ablation. Radiology. 2004 Jul;232(1):272-80.

46 Mauri G, Cova L, Ierace T, Baroli A, Di Mauro E, Pacella CM, et al. Treatment of metastatic lymph nodes in the neck from papillary thyroid carcinoma with percutaneous laser ablation. Cardiovasc Intervent Radiol. 2016 Jul;39(7):1023-30.

47 Wang L, Ge M, Xu D, Chen L, Qian C, Shi K, et al. Ultrasonography-guided percutaneous radiofrequency ablation for cervical lymph node metastasis from thyroid carcinoma. J Cancer Res Ther. 2014 Nov; 10 Suppl:C144-9.

48 Cazzato RL, Bonichon F, Buy X, Godbert Y, De Figuereido BH, Pointillart V, et al. Over ten years of single-institution experience in percutaneous image-guided treatment of bone metastases from differentiated thyroid cancer. Eur J Surg Oncol. 2015 Sep;41(9): 1247-55.
49 Bonichon F, Buy X, Godbert Y, Pointillart V, Henriques de Figueiredo B, Gangi A, et al. Local treatment of metastases from differentiated thyroid cancer. Ann Endocrinol. 2015 Feb;76(1 Suppl 1):1S40-6.

50 Zhang L, Zhou W, Zhan W, Peng Y, Jiang S, $\mathrm{Xu}$ S. Percutaneous laser ablation of unifocal papillary thyroid microcarcinoma: utility of conventional ultrasound and contrast-enhanced ultrasound in assessing local therapeutic response. World J Surg. 2018 Aug; 42(8):2476-84.

51 Li J, Liu Y, Liu J, Qian L. Ultrasound-guided percutaneous microwave ablation versus surgery for papillary thyroid microcarcinoma. Int J Hyperthermia. 2018 Jul;34(5):653-9.

52 Lan Y, Jin Z, Zhang Y, Song Q, Xiao J, Yan L, et al. Factors associated with health-related quality of life in papillary thyroid microcarcinoma patients undergoing radiofrequency ablation: a cross-sectional prevalence study. Int J Hyperthermia. 2020 Jan;37(1):1174-81.

53 Wang L, Xu D, Yang Y, Li M, Zheng C, Qiu $\mathrm{X}$, et al. Safety and efficacy of ultrasoundguided percutaneous thermal ablation in treating low-risk papillary thyroid microcarcinoma: a pilot and feasibility study. J Cancer Res Ther. 2019 Oct;15(7):1522-9.

54 Xiao J, Zhang Y, Zhang M, Lan Y, Yan L, Luo $\mathrm{Y}$, et al. Ultrasonography-guided radiofrequency ablation versus surgery for the treatment of solitary T1bN0M0 papillary thyroid carcinoma: a comparative study. Clin Endocrinol. 2021 Apr;94(4):684-91.

55 Jeon MJ, Kim WG, Chung KW, Baek JH, Kim WB, Shong YK. Active surveillance of papillary thyroid microcarcinoma: where do we stand? Eur Thyroid J. 2019 Dec;8(6):298-306.

56 Kim HI, Jang HW, Ahn HS, Ahn S, Park SY, Oh YL, et al. High serum TSH level is associated with progression of papillary thyroid microcarcinoma during active surveillance. J Clin Endocrinol Metab. 2018 Feb;103(2): 446-51.

57 Papini E, Guglielmi R, Gharib H, Misischi I, Graziano F, Chianelli M, et al. Ultrasoundguided laser ablation of incidental papillary thyroid microcarcinoma: a potential therapeutic approach in patients at surgical risk. Thyroid. 2011;21(8):917-20.

58 Zhang M, Tufano RP, Russell JO, Zhang Y, Zhang Y, Qiao Z, et al. Ultrasound-guided radiofrequency ablation versus surgery for lowrisk papillary thyroid microcarcinoma: results of over 5 years' follow-up. Thyroid. 2020 Mar;30(3):408-17.

59 Zhou W, Ni X, Xu S, Zhang L, Chen Y, Zhan W. Ultrasound-guided laser ablation versus surgery for solitary papillary thyroid microcarcinoma: a retrospective study. Int J Hyperthermia. 2019 Jan;36(1):897-904.

60 Shen K, Xue S, Xie Y, Wang H, Li J, Sun Y, et al. Comparison of thermal ablation and routine surgery for the treatment of papillary thyroid microcarcinoma: a systematic review and meta-analysis. Int J Hyperthermia. 2020 Jan;37(1):913-24.
61 Guan SH, Wang H, Teng DK. Comparison of ultrasound-guided thermal ablation and conventional thyroidectomy for benign thyroid nodules: a systematic review and meta-analysis. Int J Hyperthermia. 2020 Jan;37(1):442-9.

62 Hay ID, Lee RA, Kaggal S, Morris JC, Stan MN, Castro MR, et al. Long-term results of treating with ethanol ablation 15 adult patients with cT1aN0 papillary thyroid microcarcinoma. J Endocr Soc. 2020;4(11):1-9.

63 Kwon H, Oh H-S, Kim M, Park S, Jeon MJ, Kim WG, et al. Active surveillance for patients with papillary thyroid microcarcinoma: a single center's experience in Korea. J Clin Endocrinol Metab. 2017 Jun;102(6):1917-25.

64 Tuttle RM, Fagin JA, Minkowitz G, Wong RJ, Roman B, Patel S, et al. Natural history and tumor volume kinetics of papillary thyroid cancers during active surveillance. JAMA Otolaryngol Head Neck Surg. 2017 Oct; 143(10):1015-20

65 Oda H, Miyauchi A, Ito Y, Yoshioka K, Nakayama A, Sasai $\mathrm{H}$, et al. Incidences of unfavorable events in the management of low-risk papillary microcarcinoma of the thyroid by active surveillance versus immediate surgery. Thyroid. 2016 Jan;26(1):150-5.

66 Park KW, Shin JH, Han BK, Ko EY, Chung $\mathrm{JH}$. Inoperable symptomatic recurrent thyroid cancers: preliminary result of radiofrequency ablation. Ann Surg Oncol. 2011 Sep; 18(9):2564-8.

67 Al-Salameh A, Baudry C, Gautier JF, Toubert ME, Bihan $\mathrm{H}$, Cohen R. Late liver metastasis of medullary thyroid cancer with low calcitonin levels: successfully cured by radiofrequency. Endokrynol Pol. 2016;67(3):326-9.

68 Biamonte E, Solbiati L, Ierace T, Colombo P, Lavezzi E, Mazziotti G, et al. Medullary thyroid carcinoma treated with percutaneous ultrasound-guided radiofrequency ablation. Endocrine. 2019 Sep;65(3):515-9.

69 Ha SM, Sung JY, Baek JH, Na DG, Kim J-H, Yoo H, et al. Radiofrequency ablation of small follicular neoplasms: initial clinical outcomes. Int J Hyperthermia. 2017 May;33(8):931.

70 Dobrinja C, Bernardi S, Fabris B, Eramo R, Makovac P, Bazzocchi G, et al. Surgical and pathological changes after radiofrequency ablation of thyroid nodules. Int J Endocrinol. 2015;2015:576576.

71 Schuff KG. Management of recurrent/persistent papillary thyroid carcinoma: efficacy of the surgical option. J Clin Endocrinol Metab. 2011 Jul;96(7):2038-9.

72 Lewis BD, Hay ID, Charboneau JW, McIver B, Reading CC, Goellner JR. Percutaneous ethanol injection for treatment of cervical lymph node metastases in patients with papillary thyroid carcinoma. AJR Am J Roentgenol. 2002 Mar;178(3):699-704. 
73 Hay ID, Lee RA, Davidge-Pitts C, Reading CC, Charboneau JW. Long-term outcome of ultrasound-guided percutaneous ethanol ablation of selected "recurrent" neck nodal metastases in 25 patients with TNM stages III or IVA papillary thyroid carcinoma previously treated by surgery and 131I therapy. Surgery. 2013 Dec;154(6):1448-54.

74 Guenette JP, Monchik JM, Dupuy DE. Imageguided ablation of postsurgical locoregional recurrence of biopsy-proven well-differentiated thyroid carcinoma. J Vasc Interv Radiol. 2013 May;24(5):672-9.

75 Papini E, Bizzarri G, Bianchini A, Valle D, Misischi I, Guglielmi R, et al. Percutaneous ultrasound-guided laser ablation is effective for treating selected nodal metastases in papillary thyroid cancer. J Clin Endocrinol Metab. 2013 Jan;98(1):E92-7.

76 Kim JH, Yoo WS, Park YJ, Park DJ, Yun TJ, Choi SH, et al. Efficacy and safety of radiofrequency ablation for treatment of locally recurrent thyroid cancers smaller than $2 \mathrm{~cm}$. Radiology. 2015 Sep;276(3):909-18.

77 Zhao Q, Tian G, Kong D, Jiang T. Meta-analysis of radiofrequency ablation for treating the local recurrence of thyroid cancers. J Endocrinol Invest. 2016 Aug;39(8):909-16.

78 Suh CH, Baek JH, Choi YJ, Lee JH. Efficacy and safety of radiofrequency and ethanol ablation for treating locally recurrent thyroid cancer: a systematic review and meta-analysis. Thyroid. 2016 Mar;26(3):420-8.

79 Choi Y, Jung SL, Bae JS, Lee SH, Jung CK, Jang J, et al. Comparison of efficacy and complications between radiofrequency ablation and repeat surgery in the treatment of locally recurrent thyroid cancers: a single-center propensity score matching study. Int J Hyperthermia. 2019 Jan;36(1):359-67.
80 De Baère T, Aupérin A, Deschamps F, Chevallier $\mathrm{P}$, Gaubert $\mathrm{Y}$, Boige V, et al. Radiofrequency ablation is a valid treatment option for lung metastases: experience in 566 patients with 1037 metastases. Ann Oncol. 2015;26(5): 987-91.

81 Dupuy DE, Liu D, Hartfeil D, Hanna L, Blume JD, Ahrar K, et al. Percutaneous radiofrequency ablation of painful osseous metastases: a multicenter American College of Radiology Imaging Network trial. Cancer. 2010 Feb 15;116(4):989-97.

82 Callstrom MR, Dupuy DE, Solomon SB, Beres RA, Littrup PJ, Davis KW, et al. Percutaneous image-guided cryoablation of painful metastases involving bone: multicenter trial. Cancer. 2013 Mar;119(5):1033-41.

83 Pacella CM, Di Stasio E, Guglielmi R, Baroli A, Pedrazzini L, Misischi I, et al. Role of laser ablation in multimodal treatment of radioiodine- refractory bone metastases of thyroid cancer: a retrospective study. Endocrine. 2020 Nov;70(2):338-47.

84 Auloge P, Cazzato RL, Rousseau C, Caudrelier J, Koch G, Rao P, et al. Complications of percutaneous bone tumor cryoablation: a 10 year experience. Radiology. 2019 May;291(2): 521-8.

85 Luigi Cazzato R, Auloge P, De Marini P, Rousseau C, Chiang JB, Koch G, et al. Percutaneous image-guided ablation of bone metastases: local tumor control in oligometastatic patients. Int J Hyperthermia. 2018 Dec; 35(1):493-9.

86 Cazzato RL, Garnon J, Caudrelier J, Rao PP, Koch G, Gangi A. Low-power bipolar radiofrequency ablation and vertebral augmentation for the palliative treatment of spinal malignancies. Int J Hyperthermia. 2018 Nov; 34(8):1282-8
87 Barat M, Tselikas L, de Baère T, Gravel G, Yevich S, Delpla A, et al. Thermal-ablation of vertebral metastases prevents adverse events in patients with differentiated thyroid carcinoma. Eur J Radiol. 2019 Oct;119:108650.

88 Shyn PB, Mauri G, Alencar RO, Tatli S, Shah $\mathrm{SH}$, Morrison PR, et al. Percutaneous imaging-guided cryoablation of liver tumors: predicting local progression on 24-hour MRI. AJR Am J Roentgenol. 2014 Feb;203(2): W181-91.

89 Han K, Kim JH, Yang SG, Park SH, Choi H-K, Chun S-Y, et al. A single-center retrospective analysis of periprocedural variables affecting local tumor progression after radiofrequency ablation of colorectal cancer liver metastases. Radiology. 2021;298(1):212-8.

90 Wertenbroek MWJLAE, Links TP, Prins TR, Plukker JTM, Van Der Jagt EJ, De Jong KP. Radiofrequency ablation of hepatic metastases from thyroid carcinoma. Thyroid. 2008 Oct;18(10):1105-10.

91 Yoon JH, Jeon MJ, Kim M, Ram Hong A, Kim HK, Shin DY, et al. Unusual metastases from differentiated thyroid cancers: a multicenter study in Korea. PLoS One. 2020 Aug 25;15(8): e0238207.

92 Hegedüs L, Frasoldati A, Negro R, Papini E. European thyroid association survey on use of minimally invasive techniques for thyroid nodules. Eur Thyroid J. 2020 Jul;9(4):194204

93 Papini E, Monpeyssen H, Frasoldati A, Hegedüs L. 2020 European thyroid association clinical practice guideline for the use of image-guided ablation in benign thyroid nodules. Eur Thyroid J. 2020 Jul;9(4):172-85.
ETA-CIRSE Guideline on MIT in

Malignant Thyroid Lesions
Eur Thyroid J 2021;10:185-197

DOI: $10.1159 / 000516469$ 